\title{
Measurements of CTDI and DLP from CT Image Data for Paediatrics Radiological Performance Evaluation and dose Optimisation in Ghana
}

\author{
T. A Sackey ${ }^{1,2}$, C. Schandorf ${ }^{1}$, E Raymond $^{3}$, I. Shirazu ${ }^{1}$ \\ ${ }^{1}$ Ghana Atomic Energy Commission, Kwabenya-Accra, Ghana \\ ${ }^{2}$ National Centre for Radiotherapy and Nuclear Medicine Department, Accra, Ghana \\ ${ }^{3}$ Department of Physics, University of Cape Coast, Cape Coast
}

\begin{abstract}
Risk of developing cancer in paediatric patients is higher compared with adults and hence need for optimization strategies in paediatric medical imaging is very critical. The higher risk is attributable to the fact that children have developing organs and tissues which are more sensitive to the effects of radiation, and also they have longer life expectancy which allows more time for any harmful effects of radiation to manifest. Optimization of radiological protection is a means of adjusting imaging parameters and instituting protective measures such that required images are obtained with lowest possible radiation dose, and net benefit is maximized to maintain sufficient image quality for diagnostic purposes. Special consideration is given to the availability of dose reduction measures for paediatric imaging equipment. A unique aspect of paediatric imaging is with regards to the wide range in patient sizes and weights, therefore requiring special attention to optimization and modification of equipment, technique, and imaging parameters. Good radiographic technique for paediatrics include attention to patient positioning, field size and adequate collimation, use of protective shielding, optimization of exposure factors etc. In CT, dose reduction is optimized by the adjustment of scan parameters such as mA, $\mathrm{kVp}$, and pitch in accordance with patient weight, age, region scanned, and study indication. Paediatric radiological imaging should therefore be performed by trained and experienced health personnel in the medical imaging department. The overall aim of the research was to enhance the capability of Ghana to improve the efficiency of existing modalities for paediatric medical imaging and to implement and enhance optimization techniques and methodologies for advanced paediatric medical imaging in CT. In addition to providing appropriate clinical recommendation for clinicians for dose management during CT scan. MVL DICOM application software was used to access image data during abdominal CT scan. Effective dose estimates were estimated as developed by ICRP 103 recommendations. The data collection was based on retrospective extraction of image data, using MVL platform where detailed information of the CTDIvol and DLP were available for recording. The weighted CTDI (CTDIw) was estimated by multiplying the volumetric CTDI (CTDIvoL) by the pitch factor. The effective dose was estimated by the product of the region-specific normalizing constant and the dose length product on each image. For image quality Signal to Noise Ratio was estimated and compare with effective dose for dose optimisation. In conclusion, the mean dose parameters exceeded the recommended dose parameter and hence an urgent need for an action to minimise radiation dose to paediatric patients.
\end{abstract}

Keywords : Paediatric Imaging, Radiation Dose, Dose Optimisation, MVL, Image Data

\section{INTRODUCTION}

Risk of developing cancer in paediatric patients is higher compared with adults and hence need for optimization strategies in paediatric medical imaging is very critical. The higher risk is attributable to the fact that children have developing organs and tissues which are more sensitive to the effects of radiation, and also they have longer life expectancy which allows more time for any harmful effects of radiation to manifest. Optimization of radiological protection is a means of adjusting imaging parameters and instituting protective measures such that required images are obtained with lowest possible radiation dose, and net benefit is maximized to maintain sufficient image quality for diagnostic purposes. Special consideration is given to the availability of dose reduction measures for paediatric imaging equipment. A unique 
aspect of paediatric imaging is with regards to the wide range in patient sizes and weights, therefore requiring special attention to optimization and modification of equipment, technique, and imaging parameters. Good radiographic technique for paediatrics include attention to patient positioning, field size and adequate collimation, use of protective shielding, optimization of exposure factors etc. In CT, dose reduction is optimized by the adjustment of scan parameters such as $\mathrm{mA}, \mathrm{kVp}$, and pitch in accordance with patient weight, age, region scanned, and study indication. Paediatric radiological imaging should therefore be performed by trained and experienced health personnel in the medical imaging department In x-ray computed tomography (CT) imaging large number of photons particle and energy fluence (increase $\mathrm{mAs}$ ) are absorbed to produce clearer images to enable clinical interpretation. However, introducing larger photons will increase the SNR of the image (improve image quality), where the signals are stronger with less noisy in the image. In addition, the large photons will increase the amount of photon

interaction with the body tissues hence increase possible dose deposits. Therefore the use of high $\mathrm{kV}$ and $\mathrm{mAs}$ will increase the number of photons and radiation dose to patients and hence increasing the SNR respectively. In CT imaging dose to patients are estimated from image data by using CTDIV and DLP values. Furthermore from the recorded in data, effective dose are estimated by using the product of the region-specific normalizing constant (EDLP) and the dose length product (DLP) from ICRP publication 103 [1].

In addition to the dose input parameters ( $\mathrm{mAs}$ and $\mathrm{kVp}$ ), pitch factor also plays an important role in dose management. Where, increasing the pitch factor technically reduces radiation organ dose, but practically other factors must be considered. While increasing the pitch factor reduces the dose if all other factors are the same, which also affects image quality. The pitch factor affect image quality and patient dose in two folds. First, the pitch can place a limit on the maximum detail or spatial resolution that can be obtained in the axial slice thickness direction. Second, increasing pitch will increase image noise. However, most CT systems operates with automatic exposure control (AEC) system, that automatically increases the $\mathrm{mAs}$ and dose to maintain a specific noise level as other factors including slice thickness, matrix size, field of view and pitch are changed. An advantage of increasing pitch is to reduce scanning time, not to reduce dose. The appropriate action is to select pitch factor values that provide a balance among the image quality and scan time requirements and concerns for patient exposure. This comes from experience and the use of existing national or international guidelines and appropriate references, mostly based on Basic Safety Standard (BSS) by IAEA. To achieve this IAEA offers regional and international training courses to member countries. In particular in the field of optimization and image quality, these courses are catered for radiologists, medical physicists, radiographers and other medical professionals. The goal of medical physicist in the team is to establish quality control practices, provide measuring methodology and support optimization processes for patients', clinicians and general public safety [2]. The Agency's training services can, through a structured process, address the needs of individual professionals (through fellowships and scientific visits) and, via national training courses of Member States.

\section{OBJECTIVES}

The aim of this study is to improve the efficiency of CT imaging for paediatric medical imaging. It is also to suggest implementation and enhancement of dose optimization techniques and methodologies for advanced paediatric medical imaging in CT for appropriate paediatric dose optimisation in Ghana.

\section{EXPOSURE AND DOSE PARAMETERS}

It is important to note that photons are energetic enough to overcome the binding energy of an orbiting electrons in an atoms. This energetic photon can knock off electron from its orbital shell with an angle of 180 degrees, thereby creating ions. In human body the result of this knock off when exposed to photons, results in the creation of hydroxyl radicals in the body $[3,4]$. These are due to the $\mathrm{x}$-ray interactions with the human body cells which consist of approximately $70 \%$ water molecules. The nearby DNA will cause a base damage or strand breaks and the hydroxyl may even ionize DNA directly. It should be noted that, various systems within the cell may rapidly 
repair most of these radiation-induced damage, this however is based on a number of factors. However, it is less easy to repaired double-strand breaks, which may lead to induction of cancer [4]. These biological exposure to photon energy give rise to the determination of various fundamental dosimetric quantity in radiological imaging. In medical imaging where low-dose radiation exposure is use, the risk-related quantities can be obtained from the practical dosimetric quantities. These quantities are express from CTDIW and DLP, using the doseconversion coefficients in Table 1 for specific organ dose and regional effective dose respectively. These quantities are obtained as part of the image data and on the console. In addition, specific organ dose and regional effective dose are general estimated for referencing and provide advice to clinicians.

Table 1. Region specific normalized effective doses for CT examination

\begin{tabular}{|c|c|c|c|c|c|}
\hline $\begin{array}{l}\mathrm{CT} \\
\text { examinations }\end{array}$ & $\begin{array}{l}\text { Effective } \\
\text { Dose mSv }\end{array}$ & $\begin{array}{l}\text { DLP } \\
\mathrm{mGy} \\
\mathrm{cm}\end{array}$ & $\begin{array}{l}\text { CTDI } \\
\mathrm{mGy}\end{array}$ & $\begin{array}{l}\text { CTDIVoL } \\
\text { mGy }\end{array}$ & $\begin{array}{l}\text { EDLP } \\
\mathrm{mSv} \mathrm{mGy}^{1} \mathrm{~cm}^{-1}\end{array}$ \\
\hline Head CT & $1-2$ & 1050 & 60 & 73.80 & 0.0023 \\
\hline Chest CT & $5-7$ & 650 & 30 & 36.90 & 0.017 \\
\hline Pelvis CT & $3-4$ & 570 & 35 & 43.05 & 0.019 \\
\hline $\begin{array}{l}\text { Abdomen } \\
\text { CT }\end{array}$ & $5-7$ & 780 & 35 & 43.05 & 0.0153 \\
\hline $\begin{array}{l}\text { Abdomen- } \\
\text { Pelvis CT }\end{array}$ & $8-14$ & & & & 0.015 \\
\hline
\end{tabular}

An important dose parameter of interest is the effective dose. It is defined as the sum of the weighted equivalent doses in all the tissues and organs of the body. A broad estimates of effective dose (E) may be derived from values of DLP for an examination using appropriately normalized coefficients (Table 1) designed by ICRP and European commission [7]. The effective dose is define as the product of the region-specific normalizing constant (EDLP) and the dose length product (DLP).

Define mathematically as:

$$
\mathrm{E}=\mathrm{EDLP}{ }^{*} \mathrm{DLP}
$$

This definition by ICRP was used in this study to estimate the effective dose with known DLP and EDLP [8]. However, the estimate of the effective dose is useful when comparing doses from different diagnostic procedures and for comparing the use of similar technologies and procedures in different hospitals and countries as well as the use of different technologies for the same medical examination. Therefore these values are purely for purposes of comparism but not for estimating dose to individual patients.

Furthermore, in the process of dose optimisation a balance between the dose to patients and the ability of a clinician to answer clinical questions is essential, especially in paediatric imaging. Where there is the high potential of cancer induction in the future.

\section{METHODOLOGY}

The DICOM application software standard supplement was issued in 2007 for the reporting of dose parameters in CT [9]. This became mandatory for all manufacturers of CT equipment. It requires a report summary to be given for the whole patient examination and the accumulated dose applied. The patient information, the patient study information and the general equipment information is stored within the general part of the structured report. This development enable the obvious difficulties in measuring the distribution of dose within the body during CT imaging to be overcome. A more practical dosimetric quantities captured as part of image data were used to readily estimate these parameters from closely related measurements. The risk-related quantities were obtained from the practical input dosimetric quantities CTDI and DLP which led to the estimate of effective dose, using the dose-conversion coefficients in Table 1.

On the image data, using MVL platform detail information of the CTDIvol and DLP were available for recording. On the same platform the mean and standard deviation of region of interest drawn on the images were recorded on each of the various regions.

Effective dose using CTDIw and DLP

The weighted CTDI (CTDIW) was estimated by multiplying the volume CTDI (CTDIVOL) by the pitch factor expressed mathematically as:

$C T D I_{W}=\mathbf{p} C T D I_{V O L}$

Where $\mathrm{p}$ is the pitch factor and varies from $0.813-1.0$ is the average pitch factor of the scanning protocol. 
The CTDIW and DLP enable organ and effective dose to be estimated using equation 1 and 2 with recommended ICRP region-specific normalized effective dose coefficient in Table 1. Hence, broad estimates of effective dose (E) were derived from values of DLP and CTDIVOL respectively, for each examination using the appropriately normalized coefficients.

To estimate the various effective dose, DLP and regionspecific normalizing constant or DLP conversion factor (EDLP) as developed by ICRP Publication 103 were used and define as:

$\mathbf{E}=\mu \boldsymbol{D L P}$.

where $\mu$ is $0.0153,0.017,0.019$ and 0.0023 for Abdomen, Chest, Pelvis and Head region-specific normalizing constant respectively were used to estimate effective dose based on ICRP publication 103 as stated. This is because the effective dose is NOT measured but it is a theoretical calculated dose based on the organs exposed by the applied radiation multiplied by tissue-weighting factors. Because the tissue-weighting factors can change with new data and continuing analysis of existing data with better analytical tools, the effective dose conversion factor estimates can change over time

\section{RESULTS}

These are presented in Table 2 to 5 for head, chest, abdomen and pelvis respectively

Table 2 : Summary of Head Parameters

\begin{tabular}{|r|r|r|r|r|r|r|}
\hline Head & Age & comments & DLP & CTDI $_{\mathrm{V}}$ & Effective Dose & SNR \\
\hline Mean & $5 \mathrm{Y}$ & - & 1214.0 & 67.1 & 2.79 & 8.22 \\
\hline Max & $18 \mathrm{Y}$ & TRUAMA & 5399.9 & 226 & 12.42 & 13.40 \\
\hline Min & $1 \mathrm{D}$ & - & 30.2 & 1.5 & 0.07 & 1.149 \\
\hline
\end{tabular}

Table 3: Summary of Chest Parameters

\begin{tabular}{|r|r|r|r|r|r|r|}
\hline Chest & Age & Comments & DLP & CTDIV & Effective Dose & SNR \\
\hline Mean & $12 \mathrm{Y}$ & & 327.7 & 24 & 5.57 & \\
\hline Max & $15 \mathrm{Y}$ & PNEUMONIA & 566.7 & 99.2 & 9.63 & \\
\hline Min & $8 \mathrm{Y}$ & & 219.9 & 3.8 & 3.74 & 3.58 \\
\hline
\end{tabular}

Table 4: Abdominal Parameters

\begin{tabular}{|c|c|c|c|c|c|c|}
\hline Abdomen & Age & comments & DLP & $\mathrm{CTDI}_{\mathrm{V}}$ & Effective Dose & SNR \\
\hline Mean & $13 \mathrm{Y}$ & 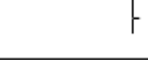 & 722.1 & 10.3 & 11.05 & 8.02 \\
\hline Max & $17 Y$ & $\begin{array}{r}\text { ABDOMINAL } \\
\text { PAIN } \\
\end{array}$ & 1311.5 & 22.2 & 20.07 & 10.49 \\
\hline Min & $8 Y$ & - & 305.1 & 4.7 & 4.67 & 5.05 \\
\hline
\end{tabular}

Table 5 : Summary of Pelvis Parameters

\begin{tabular}{|r|r|r|r|r|r|r|}
\hline Pelvis & Age & comments & DLP & CTDI $_{V}$ & Effective Dose & SNR \\
\hline Mean & $9 \mathrm{Y}$ & - & 470.2 & 10.8 & 8.93 & 9.65 \\
\hline Max & $16 \mathrm{Y}$ & INJURY & 871.4 & 19.5 & 16.56 & 12.08 \\
\hline Min & $5 \mathrm{Y}$ & - & 134 & 1.6 & 2.55 & 5.32 \\
\hline
\end{tabular}

\section{DISCUSSIONS}

It's of interest to note that increasing use of radiationbased imaging tests for repeated imaging and in young patients should be discourage unless otherwise it is extremely necessary. The risks are likely to outweigh the benefits in younger patients who are typically with very high cell sensitive, and who can likely be imaged with alternative imaging modality without the use of radiation like Ultrasound and magnetic resonance imaging. Generally CT has high radiation dose compared with chest $\mathrm{x}$-rays radiography.

In paediatric imaging optimisation is essential because it ensure that the produced image met the diagnostic request to enable all the questions to be answered. Where image noise does not distort the information for adequate and accurate analysis. This means the signal strength should not exceed the fivefold requirements that is recommended for adequate diagnostic decision. At the same time the dose to paediatric patients does not exceed the recommended values. Establishment of these procedure help to protect the patients from radiation dose which may result in a reduction of the risk to paediatric patient. This is because in an attempt to reduced dose to paediatric patients the quality of images to be produced is equally essential. That is reducing dose reduces the signal and thereby reduces the signal to noise ratio in the resulting CT image, hence, the image quality is affected. Hence it is necessary for to establish various diagnostic protocol to be followed before imaging. 
Furthermore, it's important to increase patients' awareness of their own exposure especially in young patients. Younger patients should be encouraged to keep a medical imaging history card that will allow them to track their own medical imaging history and share it with their providers all the time. So as to be access and advice on issues of radiation exposure.

In addition to improve the efficiency of CT imaging for paediatric medical imaging. A standard paediatric protocol must be made available at all the various CT Centre and more assessment must be done before a decision on imaging paediatric patients with CT. these were absent in all CT centres visited.

In conclusion there is an urgent need for most the radiology departments in Ghana to improve the radiation protection issues in the departments since it appeared that all the regional sections studied exceeded the IAEA/ICRP recommended effective dose estimates. However, the signal to noise ratio over exceeded the minimum values of five which is adequate enough to answer the clinical questions.

\section{GENERAL RECOMMENDATIONS}

In order to achieve the complete optimization strategies, there is a great demand to educate CT personnel on the effects of scan parameter settings on radiation dose to patients and image quality required for accurate diagnosis

In other to implementation and enhancement dose optimization techniques and methodologies for advanced paediatric medical imaging in CT for appropriate paediatric dose optimisation in Ghana The first is the minimization of the number of slices (hence scan length) as much as possible, without missing any vital anatomical region. This study have recommended that reduction of irradiation volume depending on specific body region being scanned, will reduce radiation dose to paediatric patients significantly.

The second is minimization of tube current (mA) based on indication of study. This study have revealed that adjustment of $\mathrm{mA}$ based on indication of study dose to patient can be reduced to $50 \%$ without significantly affecting the image quality.
The third approach is through modulation of exposure parameters (i.e., $\mathrm{kV}, \mathrm{mA}$, exposure time, and slice thickness) based on patient size and age, while maintaining a constant SNR. The study have demonstrated that by adjusting the exposure parameters based on patient age radiation doses can be reduced significantly.

\section{ACKNOWLEDGEMENT}

The Authors wish to appreciate the support received from International Atomic Energy Agency, University of Cape Coast and Radiological and Medical Sciences Research Institute of Ghana Atomic Energy Commission.

\section{REFERENCES}

[1]. DIGITAL IMAGING AND COMMUNICATIONS IN MEDICINE, Supplement 127: CT Radiation Dose Reporting (Dose SR), DICOM Standards Committee, Rosslyn, VA (2007)

[2]. Hutton B F and Osiecki A 1998 Correction of partial volume effects in myocardial SPECT J. Nucl. Cardiology. 5 Pages: 402-413

[3]. The 2007 Recommendations of the International Commission on Radiological Protection. ICRP publication 103. Ann. ICRP. 2007; 37(2-4):1-332

[4]. Aabha Dixit, IAEA Office of Public Information and Communication, IAEA Workshop to Address Challenges of Unnecessary Radiation Exposure of Patients, 15 march, 2016. Pages: 1.3

[5]. Walter Huda, Kent M. Ogden, Mohammad R. Khorasani, Converting Dose-Length Product to Effective Dose at CT, RSNA, Volume 248: Number 3 September 2008 PP 9951003

[6]. Perisinakis K, Damilakis J, Tzedakis A, Papadakis A, Theocharopoulos N, Gourtsoyannis N, 2007, "Determination of the weighted CT Dose Index in modern multi detector CT scanners", Physics in Medicine and Radiology, 2007, 52: Pages: 6485- 6495.

[7]. AAPM Report No. 39, "Specification and Acceptance Testing of Computed Tomography Scanners", 1993

[8]. Bushberg JT, et al. The Essential Physics of Medical Imaging. 3rd Ed. Section 3.3.

[9]. Hendee W R. Physics and applications of medical imaging. Rev Mod Phys. 1999; No 71: Pages: 444-50. 